Article

\title{
Investigation of Degradation Mechanism from Shear Deformation and the Relationship with Mechanical Properties, Lamellar Size, and Morphology of High-Density Polyethylene
}

\author{
Haruka Kaneyasu ${ }^{1}$, Patchiya Phanthong ${ }^{2, * \mathbb{D}}$, Hikaru Okubo ${ }^{2}$ and Shigeru Yao ${ }^{1,2} \mathbb{D}$ \\ 1 Graduate School of Chemical Engineering, Fukuoka University, 8-19-1 Nanakuma, Jonan-ku, \\ Fukuoka 814-0180, Japan; td203006@cis.fukuoka-u.ac.jp (H.K.); shyao@fukuoka-u.ac.jp (S.Y.) \\ 2 Research Institute for the Creation of Functional and Structural Materials, Fukuoka University, \\ 8-19-1 Nanakuma, Jonan-ku, Fukuoka 814-0180, Japan; hokubo@fukuoka-u.ac.jp \\ * Correspondence: patchiya@fukuoka-u.ac.jp; Tel.: +81-92-871-6631
}

check for updates

Citation: Kaneyasu, H.; Phanthong, P.; Okubo, H.; Yao, S. Investigation of Degradation Mechanism from Shear Deformation and the Relationship with Mechanical Properties, Lamellar Size, and Morphology of High-Density Polyethylene. Appl. Sci. 2021, 11, 8436. https://doi.org/ 10.3390/app11188436

Academic Editors: Mohamed Alwaeli, Jan Pizoń and Małgorzata Gołaszewska

Received: 10 August 2021

Accepted: 8 September 2021

Published: 11 September 2021

Publisher's Note: MDPI stays neutral with regard to jurisdictional claims in published maps and institutional affiliations.

Copyright: (c) 2021 by the authors. Licensee MDPI, Basel, Switzerland. This article is an open access article distributed under the terms and conditions of the Creative Commons Attribution (CC BY) license (https:/ / creativecommons.org/licenses/by/ $4.0 /)$.

\begin{abstract}
The degradation of mechanical properties is the most challenging point for the development of plastic mechanical recycling processes. Remelting and shear deformation contained in the mechanical process are a part of degradation in recycled plastics. In this study, virgin high-density polyethylene (HDPE) was simulated to be recycled by remelting and treating with shear deformation being measured at different shear treatment rates (0-100/s) using a cone-plate rheometer. The obtained shear treatment product was remolded as a thin film. The evaluation was performed comparing virgin HDPE (VPE) without any processing with shear-treated HDPE with various shear treatment rates. Tensile property, $\mathrm{X}$-ray crystallography, and morphological observations were performed in order to investigate the relationship between mechanical properties, thickness of lamellar size, and the morphology of shear-treated HDPE as compared to VPE. It can be found that the elongation at break of shear-treated HDPE at a high shear treatment rate (100/s) was significantly decreased from VPE. This degradation mechanism was related to the decreased degree of crystallinity, thickness of the crystalline layer, intermediate layer, and occurrence of crystalline orientation. This study expected to explain the degradation mechanism of HDPE from shear deformation which can be further improved by the processing conditions of the mechanical plastic recycling process.
\end{abstract}

Keywords: high-density polyethylene; shear deformation; remolding; mechanical properties; lamellar structure; crystalline lamellar morphology; intermediate layer; structure property relationship; plastic mechanical recycling

\section{Introduction}

The cumulative amount of plastic waste worldwide in 2015 was 6.3 billion tons. Among them, only 9\% was recycled and the remaining was discarded by incineration and accumulation in the environment [1]. In Japan, the total amount of waste plastic was 8.5 million tons, and polyethylene (PE) accounted for the highest amount (35.2\%) in the breakdown by resin type [2]. PE is one of the general-purpose plastics. It can be divided to high-density polyethylene (HDPE), low-density polyethylene (LDPE), and linear low-density polyethylene (LLDPE). HDPE is superior to LDPE and LLDPE in terms of heat resistance and rigidity. Furthermore, HDPE is inexpensive and easy to mold, so it is used for containers such as shampoo and detergent containers, buckets, pipes, and other personal items [2]. Single-use plastics from containers and packaging which were disposed included a large amount of HDPE. As the amount of plastic used has increased, the amount of waste has also increased which adversely has negative impacts on the environment, ecology, social, and economics [3]. Therefore, it is an urgent task to reduce the amount of waste plastic and develop alternative materials. The "Plastic Resource Recycling Strategy" was formulated by the Japanese government in 2019, and called for regulating the use of 
plastics and maximizing the recycling utilization rate [4]. It was impossible to completely eliminate the usage rate of plastics such as polyethylene and polypropylene, which are used in a variety of things in daily life and industrial applications.

One way to reduce the amount of waste plastic is to develop recycling technology and increase the recycling rate. Types of plastic recycling include chemical recycling, mechanical recycling, and thermal recycling. Mechanical recycling is the best recycling technology when considering its life cycle assessment (LCA) [5]. However, the development of recycling technology was desired due to mechanical recycling having many issues, such as the degradation of mechanical properties, contamination by foreign substances, and odors. The degradation of mechanical properties is considered to be caused by the lowering of molecular weight by chain break, cross-linking, and oxidation due to ultraviolet light or heat [6]. However, $46.8 \%$ of the total amount of waste plastic was disposable single-use plastic such as packaging and containers, and $8 \%$ was the plastic from production and processing losses [2]. For this reason, the likelihood of undergoing photodegradation due to the natural environment is extremely low. Nevertheless, the effects from the remelting and reprocessing of waste plastics in order to be recycled as pellets and the remolding process for producing plastic products also affects the degradation of properties of plastics [7].

According to our previous works, a significant relationship can be found between the mechanical properties and inner structure of recycled polypropylene remolded under different molding conditions [8]. The physical properties were changed due to the alteration of the lamellar structure from shear deformation during the recycling process. With regard to melt shear, many studies have focused on shear deformation and crystalline structure, such as flow-induced crystallization in which crystallization was accelerated by shear flow, and crystalline orientation in shear flow [9-11]. Li and Matsuba [10] studied the relationship between crystallization and the morphology of LLDPE in shear flow and the effects of relaxation time and zero-shear viscosity on precursor formation. A mechanism of precursor formation consisting of entanglement and relaxation stages was also proposed. Liu et al. [11] evaluated shear crystallization and changes in the crystalline structure of HDPE using synchrotron X-ray techniques. It was found that shear accelerated the speed of crystallization and the formation of crystalline orientation along the flow direction. Furthermore, studies of the relationships between recycled polymer materials and their mechanical properties have also implicated recycling frequency and blend ratio [12-14]. Abad et al. [12] evaluated the tensile properties of HDPE and LDPE after several extrusion cycles. It can be proved that the addition of antioxidation reduced the effect of degradation. Madi [13] found that there was a strong correlation between thermal properties and tensile behavior of the blending samples between recycled HDPE and virgin polypropylene. Many studies have already investigated shear inducement, crystalline structure, mechanical properties, and extrusion conditions, and yet the clarification of the relationships between shear history, mechanical properties, and lamellar structure has still been challenged.

This study focused on the physical degradation behavior of virgin HDPE treated with different shear treatment rates, the effect on mechanical properties, and the relationship with lamellar structures. The selection of shear treatment rates in this study was $0-100 / \mathrm{s}$. While the shear rate of the film extrusion process can be performed at 1-10,000/s [15], a shear rate which was higher than 100/s affected the distortion of the PE extrudates [16]. For this reason, the virgin HDPE was simulated to be mechanically recycled by heat and shear deformation, using a cone-plate rheometer at $0-100 / \mathrm{s}$. Then, the obtained products were remolded as thin films prior to characterization in comparison with virgin HDPE without processing. The mechanical properties were evaluated by tensile tests. X-ray crystallography such as X-ray diffraction (XRD) and small-angle X-ray scattering (SAXS) were performed to calculate the degree of crystallinity and thickness of lamellar structure. Morphological observation was carried out by transmission electron microscopy (TEM) and atomic force microscopy (AFM). Polymer chemistry confirmation was performed to evaluate the molecular weight distribution using high-temperature gel permeation chromatography (HT-GPC), and the confirmation of chemical composition was performed 
by Fourier transform infrared spectrometer (FT-IR). This study was expected to explain the physical degradation mechanism from shear deformation and the relationship between the shear deformation condition, mechanical properties, and lamellar structure of HDPE for further application in the improvement of HDPE mechanical recycling processes.

\section{Materials and Methods}

\subsection{Material}

HDPE pellets (FX201A, Film grade) were supplied by Keiyo Polyethylene Co., Ltd., Tokyo, Japan. The melt flow rate of this HDPE was $13 \mathrm{~g} / 10 \mathrm{~min}$, with $134{ }^{\circ} \mathrm{C}$ of melting temperature and $962 \mathrm{~kg} / \mathrm{m}^{3}$ of density.

\subsection{Fabrication of VPE Thin Film}

HDPE pellets were mixed with $0.3 \mathrm{wt} \%$ of an antioxidant (Irganox ${ }^{\circledR}$ B225, BASF Japan Co., Ltd., Tokyo, Japan). Then, thin film was fabricated using a press molding machine (HMM-10HP-A, Baldwin Japan Ltd., Tokyo, Japan) at $180^{\circ} \mathrm{C}, 25 \mathrm{MPa}$ for $2 \mathrm{~min}$. The thin film obtained had the dimensions of $100 \mathrm{~mm} \times 100 \mathrm{~mm}$ of width $\times$ length, and $100 \mu \mathrm{m}$ of thickness. The obtained thin film was named virgin HDPE (VPE). The schematic diagram is shown in Figure 1a.

(a)

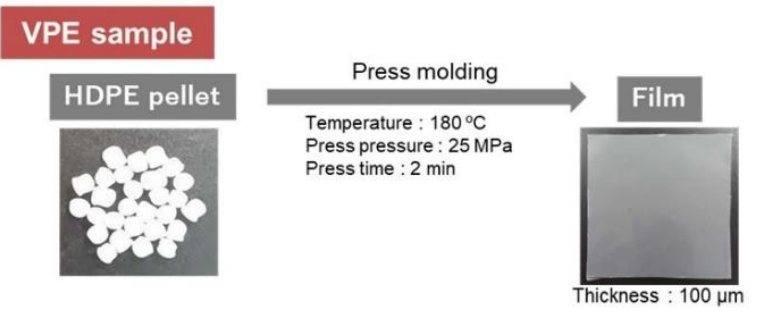

(b) Shear treatment sample

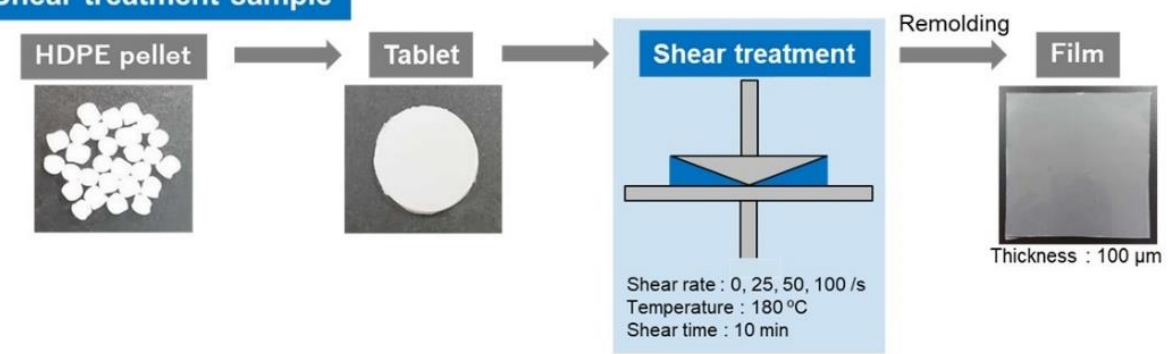

Figure 1. Schematic diagram of (a) VPE thin film fabrication; (b) shear treatment and remolding process of HDPE.

\subsection{Shear Treatment and Remolding of HDPE}

HDPE in a cylindrical tablet-shape with a thickness of $2 \mathrm{~mm}$ and diameter of $20 \mathrm{~mm}$ was prepared by hot compression of HDPE pellets containing $0.3 \mathrm{wt} \%$ of an antioxidant at $180^{\circ} \mathrm{C}, 25 \mathrm{MPa}$ for $2 \mathrm{~min}$. Shear treatment was performed using a cone-plate rheometer (Rheosol-G1000, UBM Co., Ltd., Kyoto, Japan) with different shear rates of 0-100/s at $180^{\circ} \mathrm{C}$ for $10 \mathrm{~min}$. Then, the obtained shear-treated tablet was cut and remolded as thin film with a thickness of $100 \mu \mathrm{m}$ under the same conditions as the VPE thin film fabrication. The obtained thin film was named shear-treated samples with different shear rates of $0 / \mathrm{s}$, $25 / \mathrm{s}, 50 / \mathrm{s}$, and $100 / \mathrm{s}$. A schematic diagram of the shear treatment and remolding process is identified in Figure 1b.

\subsection{Characterization}

\subsubsection{Tensile Properties}

Tensile properties of VPE and shear-treated HDPE thin films were evaluated using a small desktop testing machine (LITTLE SENSTAR LSC-02/30-2, Tokyo Koki Testing 
Machine Co., Ltd., Tokyo, Japan). The thin films were punched into a tensile test piece with the dimensions following the Japanese Industrial Standard (JIS K 71132 (1/3)). The tensile test pieces were tested with a tensile rate of $10 \mathrm{~mm} / \mathrm{min}$, which was equivalent to $0.02 \mathrm{~s}^{-1}$ of strain rate under atmospheric pressure at $25^{\circ} \mathrm{C}$ and $50 \%$ of relative humidity. The test result of each sample was averaged from the five tensile test pieces.

\subsubsection{X-ray Crystallography}

Crystalline type and degree of crystallinity of the VPE and shear-treated samples were evaluated using X-ray diffraction (XRD-6100, Shimadzu Corp., Kyoto, Japan) with a Cu $\mathrm{X}$-ray tube which operated at $40 \mathrm{kV}$ and $30 \mathrm{~mA}$. The scanning angle $(2 \theta)$ was $10^{\circ}-80^{\circ}$ with $2^{\circ} /$ min of scan speed. The degree of crystallinity was calculated from the ratio between the area under the crystalline peaks and the total area under crystalline and amorphous halo which can be determined by Equation (1).

$$
\text { Degree of crystallinity }(\%)=\frac{F_{1}}{F_{1}+F_{2}} \times 100 \%
$$

where $F_{1}$ is the area under crystalline peaks at $2 \theta=21^{\circ}$ and $24^{\circ} . F_{2}$ was indicated to the area under amorphous halo at $2 \theta=19.8^{\circ}[17,18]$.

The thicknesses of crystalline, amorphous, and intermediate layers and the degree of crystallinity were characterized by a small-angle X-ray scattering (SAXS) at the beamline BL11 of a SAGA light source (Kyushu synchrotron light research center, Saga, Japan with the proposal no. 1909068R/BL11). The beam strength was $8 \mathrm{keV}$, with an exposure time of $300 \mathrm{~s}$ and distance between sample and detector of $1140 \mathrm{~mm}$. The data analysis was carried out by the Fit2d software package, version V18.002. From the intensity $(I(q))$ and the scattering vector $(q)$, which were obtained from SAXS profile, the long period $\left(L_{0}\right)$ can be evaluated by Equation (2) [19].

$$
L_{0}=\frac{2 \pi}{q_{\max }}
$$

where $q_{\max }$ indicates the peak position of the scattering curves.

The thickness of lamellar structure and degree of crystallinity were also calculated by the electron density correlation function $[20,21]$ in Equation (3).

$$
C(z)=\int_{0}^{z_{\max }} I(q) \cdot q^{2} \cdot \cos (q \cdot z) d q
$$

where $C(z)$ is the correlation of electron density difference at a distance of $z . I(q)$ indicates the scattered intensity and $q$ identifies the scattering vector [22]. The electron density correlation function of a semi-crystalline polymer with a degree of crystallinity which was higher than $50 \%$ is shown in Figure 2a [20]. The obtained factors can be determined as the long period $\left(L_{0}\right)$, thickness of intermediate layer $\left(d_{t r}\right)$, thickness of total amorphous layer $\left(d_{\text {(Mean-Amorphous) }}\right)$ and degree of amorphousness $\left(\omega_{a}\right)$. From these factors, the thickness of total crystalline layer $\left(d_{(\text {Mean-Crystalline })}\right)$ can be further obtained by Equation (4).

$$
L_{0}=d_{(\text {Mean-Crystalline })}+d_{(\text {Mean-Amorphous })}
$$

The thickness of core amorphous layer $\left(d_{a}\right)$ was determined by the subtraction of the thickness of intermediate layer from the thickness of total amorphous layer, as shown in Equation (5).

$$
d_{a}=d_{(\text {Mean-Amorphous })}-d_{t r}
$$

A schematic diagram of the lamellar structure based on the analysis of the electron density correlation function is shown in Figure $2 b$. 


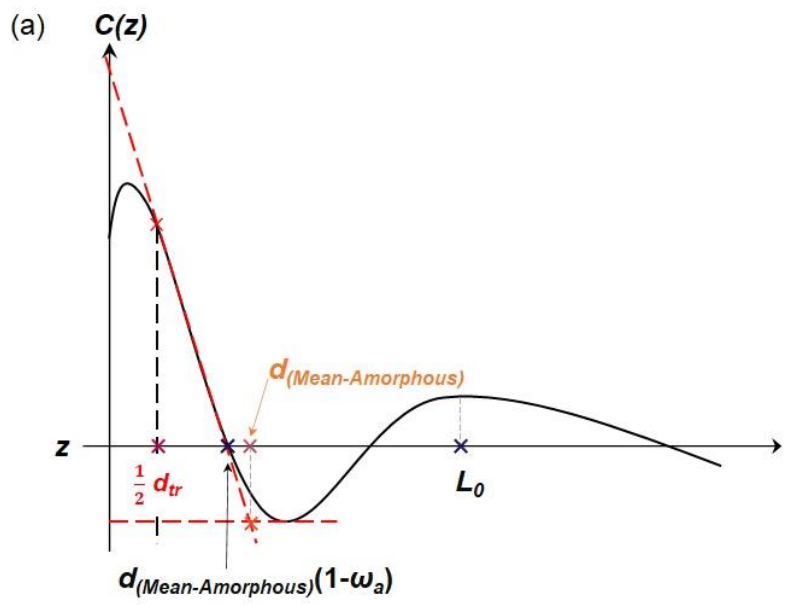

(b)

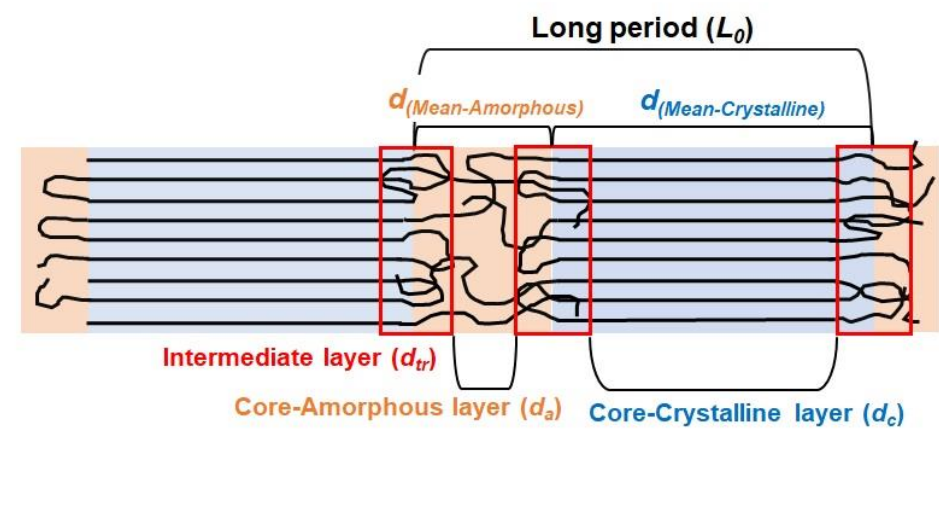

Figure 2. (a) The electron density correlation function of a semi-crystalline polymer with a degree of crystallinity higher than 50\%; (b) schematic diagram of lamellar structure based on analysis of the electron density correlation function.

\subsubsection{Morphological Observation}

The morphologies of the crystalline structure of VPE and the shear-treated samples were observed using transmission electron microscopy (TEM, JEM-2100F, JEOL Ltd., Tokyo, Japan). The thin film sample was coated with an epoxy resin and stained by $\mathrm{RuO}_{4}$ for $15 \mathrm{~min}$ prior to being cut into small pieces with a thickness of $100 \mathrm{~nm}$ using a microtome. TEM analysis was performed with an acceleration voltage of $200 \mathrm{kV}$ and at magnifications of $\times 10,000$ and $\times 40,000$.

The surface morphology was also characterized using atomic force microscopy (AFM, JPK NanoWizard ${ }^{\circledR}$ Sense AFM, Bruker Nano GmbH., Berlin, Germany). The characterization was operated under an air atmosphere in AC mode using a pyramidal cantilever with silicon coated by a metallic layer (PPP-NCHAuD, NANOSENSORSTM , Neuchâtel, Switzerland). The thin film samples were etched with $1 \mathrm{wt} \% / \mathrm{vol}$ of potassium permanganate $\left(\mathrm{KMnO}_{4}\right)$ in a ratio $2: 1$ of sulfuric acid $\left(\mathrm{H}_{2} \mathrm{SO}_{4}\right)$ and phosphoric acid $\left(\mathrm{H}_{3} \mathrm{PO}_{4}\right)$ for $2 \mathrm{~h}$. The AFM phase images were collected at $512 \times 512$ pixels.

\subsubsection{Evaluation of Molecular Weight and Chemical Composition}

The possibilities of molecular chain break and oxidative degradation of the sheartreated samples were evaluated and compared with those of the VPE. The changes of averaged molecular weight and molecular weight distribution were characterized by hightemperature gel permeation chromatography (HT-GPC, HLC-8321GPC/HT, Tosoh Corp., Tokyo, Japan). Thin film samples $(1 \mathrm{mg} / \mathrm{mL})$ were dissolved in a solution of $0.05 \mathrm{wt} \%$ of 2,6-Di-tert-butyl-p-cresol (BHT) in 1,2,4-Trichlorobenzene (TCB). Then, the sample solution was shaken at $140{ }^{\circ} \mathrm{C}$ for $1 \mathrm{~h}$. The characterization was carried out with a flow rate of $1 \mathrm{~mL} / \mathrm{min}$ at $140^{\circ} \mathrm{C}$ of temperature.

The chemical composition of shear-treated HDPE was evaluated using a Fourier transform infrared spectrometer (FT-IR, Spectrum Two ${ }^{\mathrm{TM}}$ FT-IR Spectrometer, PerkinElmer Inc., Waltham, MA, USA). The thin film was characterized with the wave number of $4500-450 \mathrm{~cm}^{-1}$ in attenuated total reflectance (ATR) mode, under room temperature.

\section{Results \& Discussion}

\subsection{Tensile Properties}

The averaged tensile properties of VPE and shear-treated HDPE at 0, 25, 50, and 100 /s are exhibited in Table 1. It can be found that the elongation at break and Young's modulus of VPE were higher than those of the shear-treated HDPE samples. Especially in the comparison between VPE and the highest shear treatment at 100/s, it can be found that elongation at break and the Young's modulus of the 100/s samples were decreased from those of VPE at $27.9 \%$ and $27.8 \%$, respectively. This suggests the degradation of the 
tensile properties of HDPE from the effects of the shear treatment and remolding processes. However, the yield stress and tensile fracture stress of the shear-treated samples were similar to VPE. Because the main application of VPE in this study was a film grade, the most important property was the elongation at break, which represents the stretching of the film. Figure 3 shows a comparison of the elongation at break between the VPE and shear-treated HDPE with different shear treatment rates. The averaged elongation at break of shear-treated HDPE at low shear rate $(0,25,50 / \mathrm{s})$ was slightly reduced from that of VPE. On the other hand, at the high shear rate of $100 / \mathrm{s}$, the averaged elongation at break was significantly decreased from VPE and the other shear-treated samples. This implies that the high shear rate had a high effect of degrading the elongation at break of the HDPE. Figure 4 shows the representative stress-strain curves of the VPE and the shear-treated HDPE at 100/s. That elongation at break of $100 / \mathrm{s}$ was obviously decreased than VPE. However, the tensile fracture stress was slightly increased from VPE.

Table 1. The averaged tensile properties of VPE and shear-treated samples.

\begin{tabular}{cccccc}
\hline Sample & $\begin{array}{c}\text { Elongation at Break } \\
{[\%]}\end{array}$ & $\begin{array}{c}\text { Toughness } \\
{\left[\mathbf{M J} / \mathbf{m}^{3}\right]}\end{array}$ & $\begin{array}{c}\text { Young's Modulus } \\
{[\mathbf{M P a}]}\end{array}$ & $\begin{array}{c}\text { Yield Stress } \\
{[\mathbf{M P a}]}\end{array}$ & $\begin{array}{c}\text { Tensile Fracture Stress } \\
{[\mathbf{M P a}]}\end{array}$ \\
\hline VPE & $2248.4 \pm 72.9$ & $350.6 \pm 14.5$ & $384.6 \pm 21.2$ & $17.5 \pm 0.8$ & $23.8 \pm 0.8$ \\
$0 / \mathrm{s}$ & $1969.0 \pm 159.2$ & $268.6 \pm 7.2$ & $268.6 \pm 22.5$ & $13.9 \pm 0.3$ & $18.7 \pm 0.3$ \\
$25 / \mathrm{s}$ & $2122.8 \pm 70.6$ & $269.7 \pm 6.1$ & $284.7 \pm 7.9$ & $13.8 \pm 0.2$ & $18.6 \pm 0.2$ \\
$50 / \mathrm{s}$ & $1951.8 \pm 398.3$ & $313.9 \pm 14.0$ & $314.5 \pm 72.3$ & $16.3 \pm 0.8$ & $22.4 \pm 0.9$ \\
$100 / \mathrm{s}$ & $1620.6 \pm 370.9$ & $342.6 \pm 6.5$ & $277.7 \pm 62.9$ & $17.5 \pm 0.3$ & $25.1 \pm 0.2$ \\
\hline
\end{tabular}

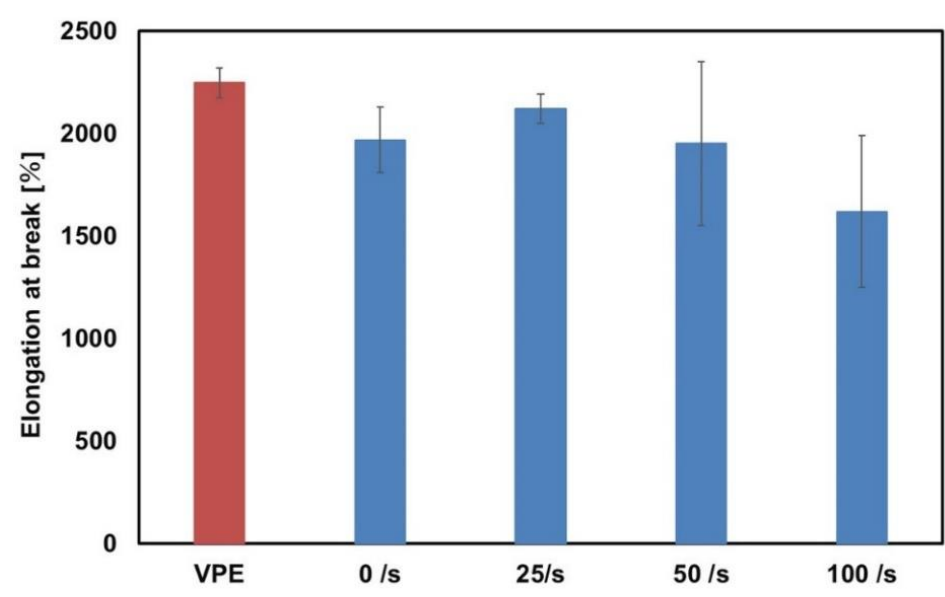

Figure 3. Elongation at break of VPE and shear-treated samples.

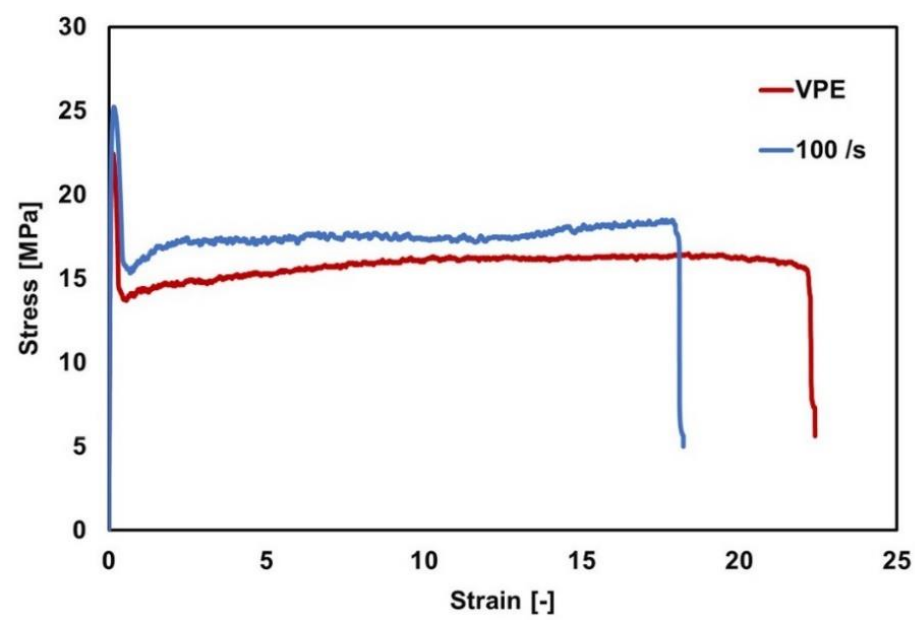

Figure 4. Representative stress-strain curves of VPE and shear-treated HDPE at 100/s. 


\subsection{X-ray Crystallography}

3.2.1. Crystalline Type and Degree of Crystallinity Evaluated by XRD

The crystalline type and degree of crystallinity of the VPE and shear-treated samples were analyzed by XRD. As shown in Figure 5, all shear-treated samples showed the same trend of XRD profiles as compared to VPE. The crystalline peaks were detected at $21.6^{\circ}$ and $24.0^{\circ}$ of $2 \theta$ indicated (110) and (200) of the PE crystalline plane [23]. From these XRD profiles, it can be inferred that shear treatment at $0-100 / \mathrm{s}$ and the remolding process had no effects on the types of crystallinity in the HDPE. For the comparison of the degree of crystallinity, shear-treated samples showed a slight decrease from the VPE with the increase of the shear treatment rate. Shear-treated samples at $100 / \mathrm{s}$, the highest shear treatment rate, showed the lowest of degree of crystallinity at $80.3 \%$. From these results, it can be concluded that the shear treatment slightly decreased the degree of crystallinity in HDPE.

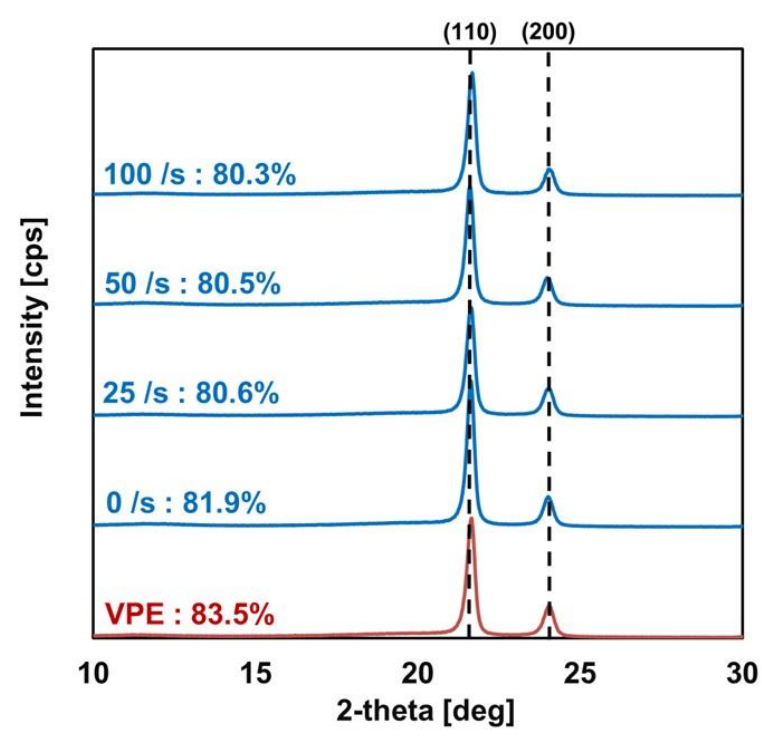

Figure 5. XRD profile and degree of crystallinity of VPE and shear-treated samples.

3.2.2. Degree of Crystallinity and Thickness of Lamellar Structure Evaluated by SAXS

Figure 6 showed the SAXS profile of VPE and shear-treated HDPE at 0-100/s. The peak position $\left(q_{\max }\right)$ of VPE was $0.257 \mathrm{~nm}^{-1}$. However, at the high shear treatment rates $(50-100 / \mathrm{s})$, the peak position shifted to high values at $0.262 \mathrm{~nm}^{-1}$ of $100 / \mathrm{s}$. This implies a decreasing of the long period $\left(L_{0}\right)$ of the $50-100 / \mathrm{s} \mathrm{samples,} \mathrm{which} \mathrm{can} \mathrm{be} \mathrm{determined} \mathrm{from}$ the peak position of the SAXS profile.

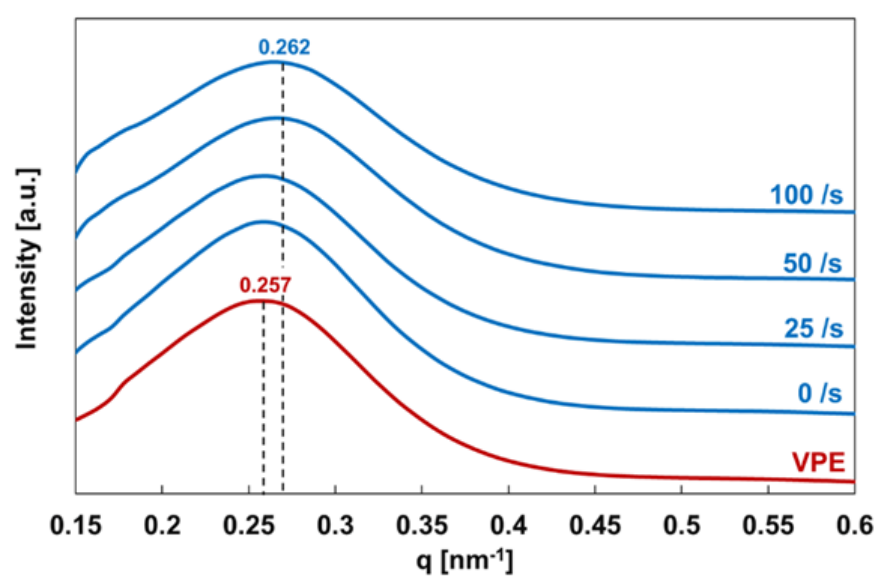

Figure 6. SAXS profiles of VPE and shear-treated samples. 
The results of $q_{\max }$ and the long period calculated from the SAXS profile (Figure 6) are summarized in Table 2. The shifting of the peak position of 100/s related to the decreasing of long period which was the combination between the thicknesses of the crystalline, amorphous, and intermediate layers, suggesting that the shear treatment rate affected the changes of thickness of the lamellar structure of the shear-treated HDPE.

Table 2. Peak position $\left(q_{\max }\right)$ of the SAXS scattering curves and the calculated long period $\left(L_{0}\right)$ of VPE and shear-treated samples.

\begin{tabular}{ccc}
\hline Sample & $\boldsymbol{q}_{\max }\left[\mathrm{nm}^{-1}\right]$ & Long Period $\left(L_{0}\right)[\mathrm{nm}]$ \\
\hline VPE & 0.257 & 24.4 \\
$0 / \mathrm{s}$ & 0.257 & 24.4 \\
$25 / \mathrm{s}$ & 0.257 & 24.4 \\
$50 / \mathrm{s}$ & 0.270 & 23.3 \\
$100 / \mathrm{s}$ & 0.262 & 23.9 \\
\hline
\end{tabular}

The degree of crystallinity and thickness of lamellar structure of the VPE and sheartreated samples were characterized by SAXS, and also analyzed by the electron density correlation function method. The results are tabulated in Table 3. The degrees of crystallinity of shear-treated HDPE with low shear rates $(0-50 / \mathrm{s})$ were similar to VPE. At the high shear rate of 100/s, the degree of crystallinity was reduced around 10\% from VPE. It can be found that the degree of crystallinity calculated by SAXS (Table 3) showed a similar trend with the calculation by XRD (Figure 5), even though the values are slightly different from the distinction of the instruments and the calculation methods. For the comparison of the long period, which is the combination of the total crystalline layer and total amorphous layer, the long period of shear-treated samples was slightly decreased from VPE. This reduction of the long period was related to the decreased thickness of core-crystalline layer, especially at the high shear treatment rate. The enlargement of the core-amorphous layer and the reduction of the core-crystalline layer were also associated with the decreasing degree of crystallinity of the shear-treated HDPE at 100/s. For the thickness of the intermediate layer, which is the interface area between the crystalline and amorphous regions, it was also shortened with the increasing of the shear treatment rate. From these calculations, it can be confirmed that shear treatment rate affected the changes to the degrees of crystallinity and thickness of lamellar structure of the HDPE. Especially at $100 / \mathrm{s}$, the degree of crystallinity, long period, core-crystalline layer, and intermediate layer were decreased; however, the core-amorphous layer was slightly increased as compared to VPE.

Table 3. Degree of crystallinity and thickness of long period, core-crystalline layer, core-amorphous layer, and intermediate layer of the VPE and shear-treated samples, evaluated by SAXS.

\begin{tabular}{cccccc}
\hline & Crystallinity & \multicolumn{4}{c}{ Thickness of Lamellar Structure [nm] } \\
\cline { 3 - 6 } Sample & & $\begin{array}{c}\text { Long Period } \\
\left(\boldsymbol{L}_{\mathbf{0}}\right)\end{array}$ & $\begin{array}{c}\text { Core-Crystalline } \\
\left(\boldsymbol{d}_{\boldsymbol{c}}\right)\end{array}$ & $\begin{array}{c}\text { Core-Amorphous } \\
\left(\boldsymbol{d}_{\boldsymbol{a}}\right)\end{array}$ & $\begin{array}{c}\text { Intermediate Layer } \\
\left(\boldsymbol{d}_{t r}\right)\end{array}$ \\
\hline $\mathrm{VPE}$ & 80.47 & 20.80 & 15.49 & 2.27 & 1.52 \\
$0 / \mathrm{s}$ & 80.49 & 20.70 & 15.53 & 2.21 & 1.48 \\
$25 / \mathrm{s}$ & 80.58 & 20.70 & 15.60 & 2.18 & 1.46 \\
$50 / \mathrm{s}$ & 79.57 & 19.80 & 14.62 & 2.30 & 1.44 \\
$100 / \mathrm{s}$ & 70.70 & 19.80 & 14.75 & 2.41 & 1.32 \\
\hline
\end{tabular}

The relationship between mechanical properties and lamellar structure was studied by correlating between each thickness with the elongation at break which was the most important property of the HDPE film grades. Figure 7a shows the correlation between the thickness of core-amorphous layer $\left(d_{a}\right)$ and elongation at break. A negative correlation can be detected. At the low shear rates $(0-50 / \mathrm{s})$, the elongation at break was higher 
and the thickness of core-amorphous layer was lower than at the high shear rate (100/s). For the correlation between the thickness of intermediate layer $\left(d_{t r}\right)$ and elongation at break (Figure $7 \mathrm{~b}$ ), a positive correlation was detected with a high correlation coefficient $\left(R^{2}=0.89\right)$. The reduction of the thickness of the intermediate layer was found in all shear-treated samples with the decrease of the elongation at break. It is worth noting that shear-treated HDPE at 100/s showed the most shortening of the thickness of the intermediate layer and elongation at break, as compared to VPE and the other shear-treated samples. This suggests that shear treatment not only affected the decreasing elongation at break, the thickness of the lamellar structure, especially in the intermediate layer, was also decreased in the HDPE.

(a)

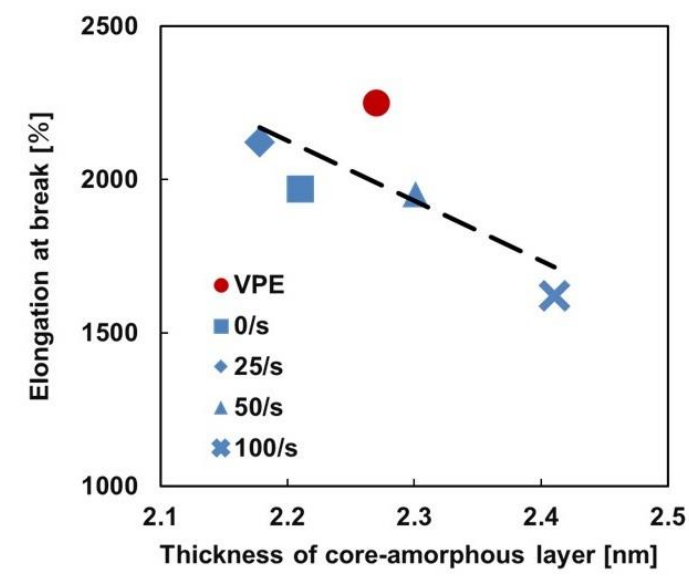

(b)

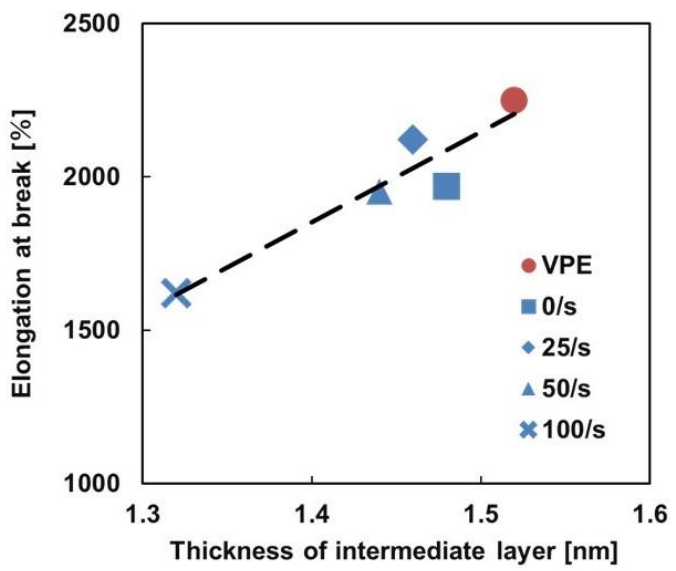

Figure 7. Relationship between elongation at break and (a) thickness of core-amorphous layer; (b) thickness of intermediate layer.

\subsection{Morphological Observation}

From having the greatest reduction of elongation at break, degree of crystallinity, thickness of long period, and intermediate layer, shear-treated HDPE at 100/s was selected to be a representative of the shear-treated samples for comparison of morphology with VPE. Figure 8 shows the TEM images of VPE and shear-treated HDPE at 100/s thin film at different magnification levels. At low magnification $(\times 10,000$, Figure $8 a)$, the morphology of the crystalline region was similar between VPE and 100/s. It is worth noting that a difference can be obviously detected in the high-magnification TEM observation $(\times 40,000$, Figure $8 b$ ). The stacking of lamellar was detected in both samples; however, the lamellar orientation was different. In case of VPE, a non-oriented lamellar structure was detected which was a general form of PE without any treatment. On the other hand, shear treatment affected the generation of the orientation of lamellar structure in shear-treated HDPE in parallel to the shear direction. These changes of lamellar orientation also affected the decreased elongation at break of the shear-treated HDPE at 100/s as compared to VPE.

AFM was also used for the characterization of morphological observations. Figure 9 shows AFM phase images of VPE (Figure 9a) and the shear-treated HDPE at 100/s (Figure $9 b)$. The bright area indicates the crystalline phase; however, the dark area defines the amorphous region. In a similar trend with TEM images (Figure 8), it was found that the non-oriented crystalline phase was detected in the VPE. However, the orientation of the crystalline region obviously occurred in shear-treated HDPE at 100/s. In addition, the generation of lamellar orientation was in parallel to the shear direction. 


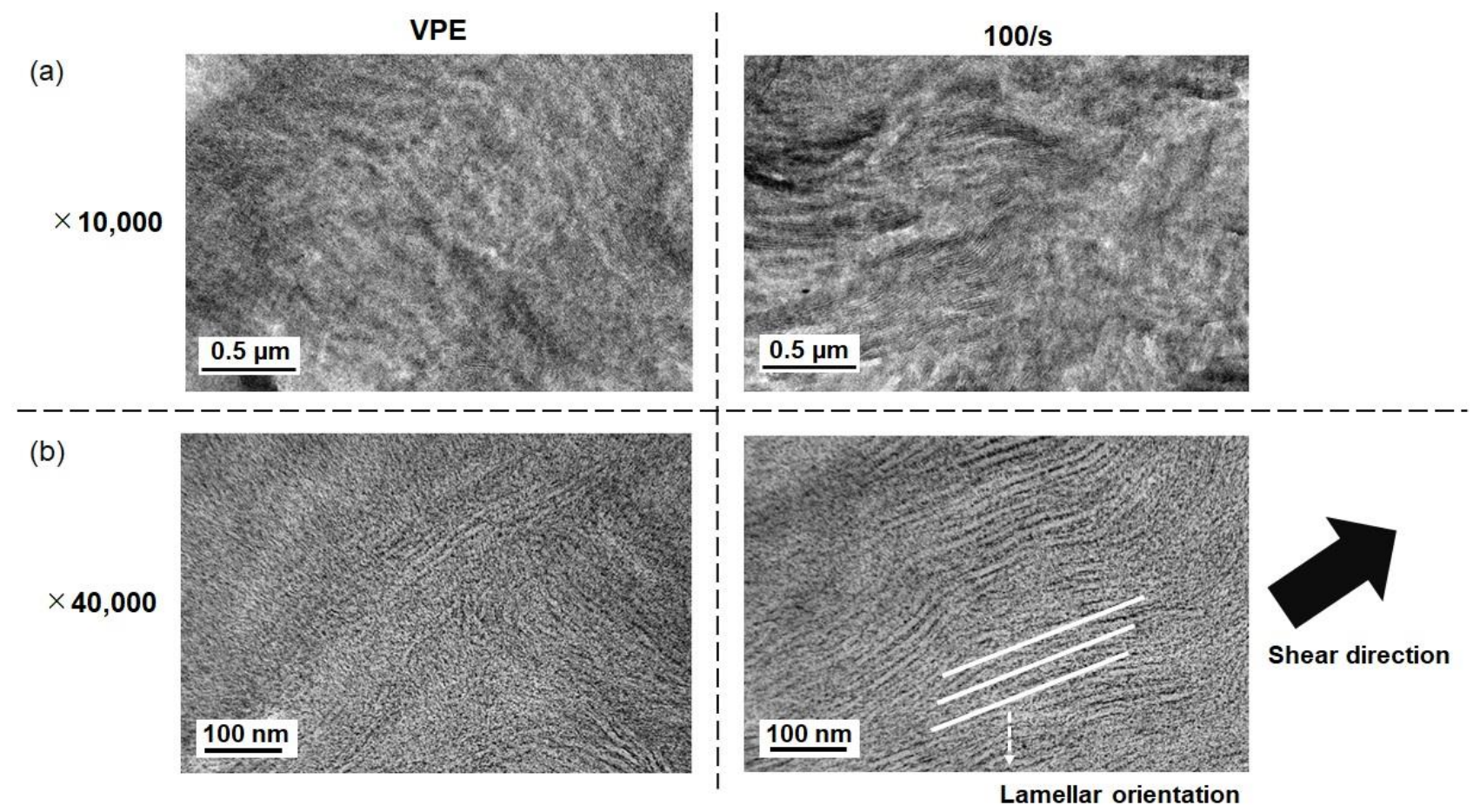

Figure 8. TEM images of VPE and shear-treated HDPE at 100/s at (a) $\times 10,000$, and (b) $\times 40,000$ magnification.
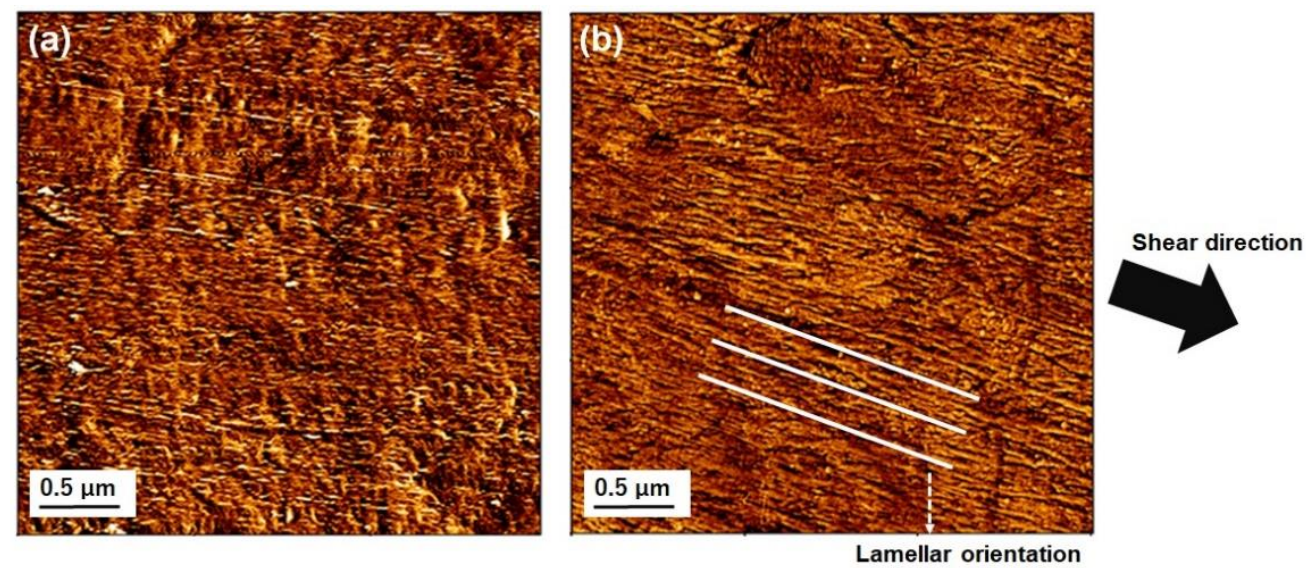

Figure 9. AFM phase images of (a) VPE; and (b) shear-treated HDPE at 100/s.

From these morphological observations by TEM and AFM, it can be confirmed that crystalline orientation was detected in the shear-treated HDPE which was affected from shear treatment. In addition, the creation of a crystalline orientation in the shear-treated samples was also related to the changes of elongation at break of HDPE.

\subsection{Evaluation of Molecular Weight and Chemical Composition}

3.4.1. Averaged Molecular Weight and Molecular Weight Distribution

HT-GPC was used for evaluation of the averaged molecular weight and molecular weight distribution of VPE and shear-treated samples. Figure 10 shows the molecular weight distribution curves of the VPE and shear-treated HDPE at 100/s. It can be found that the molecular weight distribution curves were almost the same between the VPE and the 100/s HDPE. 


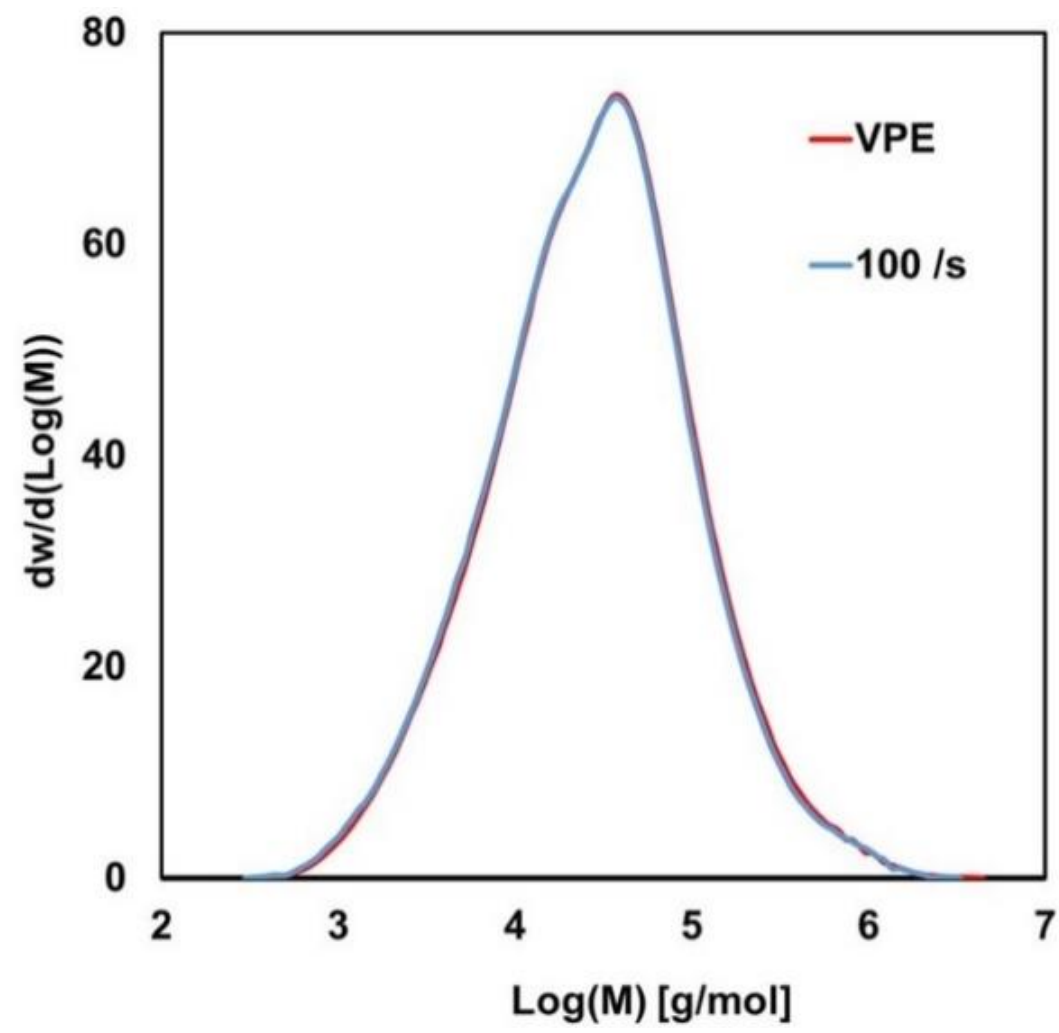

Figure 10. Molecular weight distribution curves of VPE and shear-treated HDPE at 100/s.

Table 4 shows detailed information of the number-averaged molecular weight (Mn), the weight-averaged molecular weight $(\mathrm{Mw})$, the Z-averaged molecular weight $(\mathrm{Mz})$, and the polydispersity index (Mw/Mn) of VPE and 100/s. The averaged molecular weight and polydispersity index of the shear-treated HDPE at 100/s were similar to those of the VPE. From these results, it can be confirmed that shear treatment has no effect on chain scission and no changes to the polymer chemistry of HDPE, even though the elongation at break and the thickness of lamellar structure were different.

Table 4. The averaged molecular weight (Mn, Mw, Mz) and polydispersity index (Mw/Mn) of VPE and shear-treated HDPE at $100 / \mathrm{s}$.

\begin{tabular}{ccccc}
\hline Sample & Mn $[\mathbf{g} / \mathbf{m o l}]$ & Mw $[\mathbf{g} / \mathbf{m o l}]$ & Mz $[\mathbf{g} / \mathbf{m o l}]$ & Mw/Mn \\
\hline VPE & 27,644 & 156,167 & 825,827 & 5.65 \\
$100 / \mathrm{s}$ & 26,249 & 150,914 & 786,072 & 5.75 \\
\hline
\end{tabular}

\subsubsection{Chemical Composition}

Figure 11 shows the FT-IR spectra of the VPE and the shear-treated HDPE at 100/s. The FT-IR spectra of the 100/s HDPE was similar to that of VPE. The characteristic bands of the HDPE were detected in both the VPE and the 100/s samples for C-H stretching at 2915 and $2848 \mathrm{~cm}^{-1}, \mathrm{CH}_{2}$ bending at 1472 and $1462 \mathrm{~cm}^{-1}$, and $\mathrm{CH}_{2}$ rocking at 730 and $719 \mathrm{~cm}^{-1}$ [24]. In order to confirm the changes of chemical composition after the shear treatment process, the carbonyl peak from oxidation at $1700 \mathrm{~cm}^{-1}$ [25] was focused; however, it was not detected in the shear-treated HDPE at 100/s. Therefore, no oxidative reaction occurred in the 100/s HDPE. From the results of FT-IR spectra, it can be also suggested that shear treatment has no effect on changes of chemical composition in HDPE. 


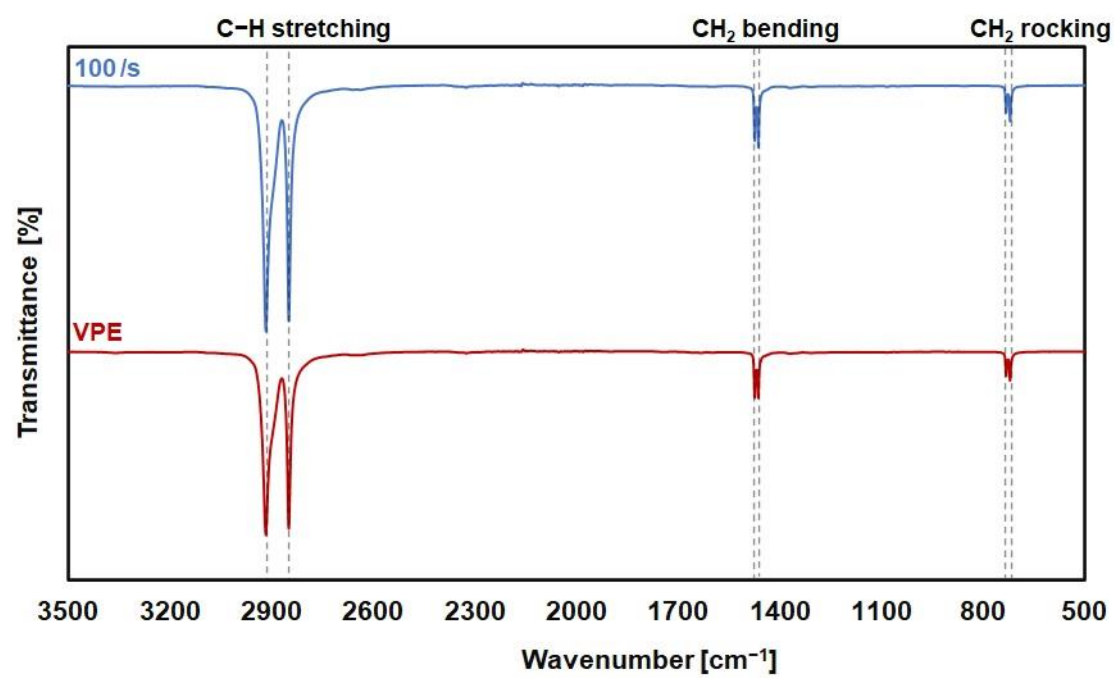

Figure 11. FT-IR spectra of VPE and shear-treated HDPE at 100/s.

\section{Conclusions}

The high shear treatment rate $(100 / \mathrm{s})$ reduced the elongation at break of HDPE compared to VPE without shear treatment. The crystalline type of shear-treated HDPE had no changes from shear deformation. However, degree of crystallinity was decreased with the shortening of long period, core-crystalline layer, and intermediate layer as compared to VPE. A positive correlation was detected between the relationship of elongation at break and the thickness of the intermediate layer. The proposed physical degradation mechanism from shear treatment is shown in Figure 12. Shear treatment at 100/s affected a decreasing of the thickness of the core-crystalline and intermediate layers, which also related to the decrease of the amount of chain entanglement and the reduction of the elongation at break [26,27]. Furthermore, shear deformation also affected the occurrence of crystalline orientation in shear-treated HDPE. With the changes of elongation at break, lamellar size, and morphology, shear-treated HDPE showed similar molecular weight distribution and chemical composition as VPE, which can prove that there were no changes in polymer chemistry from shear deformation. This study indicated that mechanical treatment affected changes to mechanical properties and lamellar structure. These findings can be further improved for development of HDPE properties and plastic mechanical recycling technology.

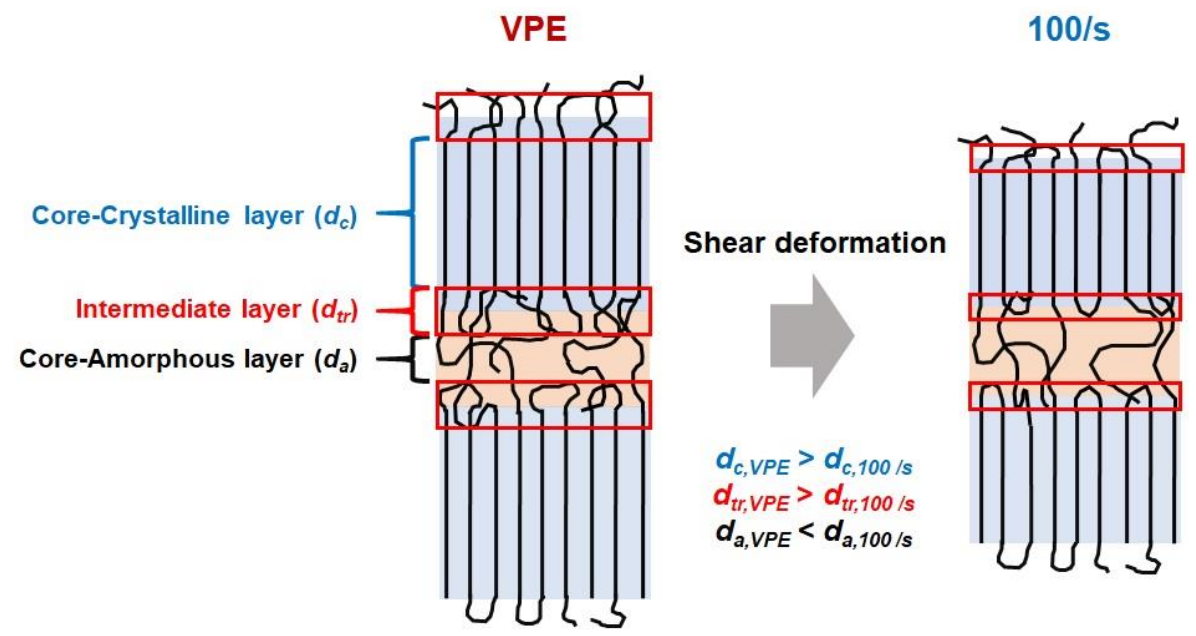

Figure 12. Proposed physical degradation mechanism of shear-treated HDPE at 100/s. 
Author Contributions: Conceptualization, P.P. and S.Y.; methodology, P.P. and S.Y.; validation, P.P. and S.Y.; formal analysis, H.K., P.P. and H.O.; investigation, H.K., P.P. and H.O.; resources, H.K., P.P. and H.O.; data curation, H.K., P.P. and H.O.; writing-original draft preparation, H.K. and P.P.; writing-review and editing, P.P.; visualization, H.K. and P.P.; supervision, S.Y.; project administration, S.Y.; funding acquisition, S.Y. All authors have read and agreed to the published version of the manuscript.

Funding: This research was funded by the New Energy and Industrial Technology Development Organization (NEDO) grant number JPNP20012, Japan.

Institutional Review Board Statement: Not applicable.

Informed Consent Statement: Not applicable.

Data Availability Statement: No new data were created or analyzed in this study. Date sharing is not applicable to this article.

Conflicts of Interest: The authors declare no conflict of interest.

\section{References}

1. Geyer, R.; Jambeck, J.R.; Law, K.L. Production, use, and fate of all plastics ever made. Sci. Adv. 2017, 3, e1700782. [CrossRef]

2. Plastic Waste Management Institute. An Introduction to Plastic Recycling. 2019. Available online: http://www.pwmi.or.jp/ei/ plastic_recycling_2019.pdf (accessed on 16 July 2021).

3. Beasmont, N.J.; Aanesen, M.; Austen, M.C.; Börger, T.; Clark, J.R.; Cole, M.; Hooper, T.; Lindeque, P.K.; Pascoe, C.; Wyles, J.R. Global ecological, social and economic impacts of marine plastic. Mar. Pollut. Bull. 2019, 142, 189-195. [CrossRef]

4. Ministry of the Environment. Resource Circulation Strategy for Plastics. Available online: https://www.env.go.jp/en/focus/jeq/ issue/pdf/Volume20January2018.pdf (accessed on 16 July 2021).

5. Dodbiba, G.; Takahashi, K.; Sadaki, J.; Fujita, T. The recycling of plastic wastes from discarded TV sets: Comparing energy recovery with mechanical recycling in the context of life cycle assessment. J. Clean. Prod. 2008, 16, 458-470. [CrossRef]

6. Briassoulis, D.; Aristopoulou, A.; Bonora, M.; Verlodt, I. Degradation characterisation of agricultural low-density polyethylene films. Biosyst. Eng. 2004, 88, 131-143. [CrossRef]

7. Ragaert, K.; Delva, L.; Geem, K.V. Mechanical and chemical recycling of solid plastic waste. Waste Manag. 2017, 69, 24-58. [CrossRef] [PubMed]

8. Tominaga, A.; Sekiguchi, H.; Nakano, R.; Yao, S.; Takatori, E. Thermal process-dependence of the mechanical properties and inner structures of pre-consumer recycled polypropylene. AIP Conf. Proc. 2015, 1664, 150011.

9. Wang, Z.; Ju, J.; Yang, J.; Ma, Z.; Liu, D.; Cui, K.; Yang, H.; Chang, J.; Huang, N.; Li, L. The non-equilibrium phase diagrams of flow-induced crystallization and melting of polyethylene. Sci. Rep. 2016, 6, 32968. [CrossRef] [PubMed]

10. Li, K.; Matuba, G. Effects of relaxation time and zero shear viscosity on structural evolution of linear low-density polyethylene in shear flow. J. Appl. Polym. Sci. 2018, 135, 46053. [CrossRef]

11. Liu, Y.; Gao, S.; Hsiao, B.S.; Norman, A.; Tous, A.H.; Throckmorton, J.; Doufas, A.; Zhang, Y. Shear induced crystallization of bimodal and unimodal high density polyethylene. Polymer 2018, 153, 223-231. [CrossRef]

12. Abad, M.J.; Ares, A.; Barral, L.; Cano, J.; Díez, F.J.; García-Garabal, S.; Ramírez, C. Effects of a mixture of stabilizers on the structure and mechanical properties of polyethylene during reprocessing. J. Appl. Polym. Sci. 2004, 92, 3910-3916. [CrossRef]

13. Madi, N.K. Thermal and mechanical properties of injection molded recycled high density polyethylene blends with virgin isotactic polypropylene. Mater. Des. 2013, 46, 435-441. [CrossRef]

14. Mourad, A.I. Thermo-mechanical characteristics of thermally aged polyethylene/polypropylene blends. Mater. Des. 2010, 31, 918-929. [CrossRef]

15. Franck, A. Understanding Rheology of Thermoplastic Polymers. TA Instruments Application Note No. AAN013. 2008. Available online: http:/ / www.tainstruments.com/pdf/literature/AAN013_V_1_U_Thermoplast.pdf (accessed on 27 August 2021).

16. Bagley, E.B.; Storey, S.H.; West, D.C. Post extrusion swelling of polyethylene. J. Appl. Polym. Sci. 1963, 7, 1661-1672. [CrossRef]

17. Gopalan, M.R.; Mandelkern, L. Degree of crystallinity of linear polyethylene from wide-angle X-ray diffraction. Polym. Lett. 1967, 5, 925-929. [CrossRef]

18. Papajani, B.; Qoku, E.; Malkaj, P.; Dilo, T. The study of phase compound and the degree of crystallinity of recycled LDPE by X-ray diffractometer and optical microscope. Int. J. Sci. Res. 2015, 4, 2228-2232.

19. Yao, G.; Duan, T.; Chi, E.; Guo, P.; Li, Y.; Wang, Z. Microbeam two-dimensional small-angle X-ray scattering investigating the effects of reduced graphene oxide on local microstructures of high-density polyethylene/reduced graphene oxide nanocomposite bars. R. Soc. Open Sci. 2019, 6, 181866. [CrossRef]

20. Strobl, G.R.; Schneider, M.J.; Voigt-Martin, I.G. Model of partial crystallization and melting derived from small-angle X-ray scattering and electron microscopic studies on low-density polyethylene. J. Polym. Sci. Polym. Phys. Ed. 1980, 18, 1361-1381. [CrossRef] 
21. Strobl, G.R.; Schneider, M. Direct evaluation of the electron density correlation function of partially crystalline polymers. J. Polym. Sci. Polym. Phys. Ed. 1980, 18, 1343-1359. [CrossRef]

22. Ryan, A.J. A direct method to determine the degree of crystallinity and lamellar thickness of polymers: Application to polyethylene. Polymer 1994, 35, 4537-4544. [CrossRef]

23. Furukawa, T.; Sato, H.; Kita, Y.; Matsukawa, K.; Yamaguchi, H.; Ochiai, S.; Siesler, H.W.; Ozaki, Y. Molecular structure, crystallinity and morphology of polyethylene/polypropylene blends studied by Raman mapping, scanning electron microscopy, wide angle X-ray diffraction, and differential scanning calorimetry. Polym. J. 2006, 38, 1127-1136. [CrossRef]

24. Jung, M.R.; Horgen, F.D.; Orski, S.V.; Rodriguez, V.C.; Beers, K.L.; Balazs, G.H.; Jones, T.T.; Work, T.M.; Brignac, K.C.; Royer, S.; et al. Validation of ATR FT-IR to identify polymers of plastic marine debris, including those ingested by marine organism. Mar. Pollut. Bull. 2018, 127, 704-716. [CrossRef] [PubMed]

25. Qin, H.; Zhang, Z.; Feng, M.; Gong, F.; Zhang, S.; Yang, M. The influence of interlayer cations the photo-oxidative degradation of polyethylene/montmorillonite composite. J. Polym. Sci. B Polym. Phys. 2004, 42, 1127-1136. [CrossRef]

26. Yadegari, A.; Morshedian, J.; Khonakdar, H.A.; Wagenknecht, U.; Heinrich, G.; Malanin, M.; Eichhorn, K.J.; Fischer, D. Correlation of crystal alignment with interphase content in oriented high density polyethylene cast films. CrystEngComm 2016, 18, 2337-2346. [CrossRef]

27. Yadegari, A.; Morshedian, J.; Khonakdar, H.A.; Wagenknecht, U.; Heinrich, G. Influence of precursor film microstructure on properties of HDPE microporous membranes prepared by stretching. J. Appl. Polym. Sci. 2017, 134, 44725. [CrossRef] 